Article

\title{
Counterintuitive Test Problems for Distance-Based Similarity Measures Between Intuitionistic Fuzzy Sets
}

\author{
Hui-Chin Tang *(1) and Shen-Tai Yang * \\ Department of Industrial Engineering and Management, National Kaohsiung University of Science and \\ Technology, Kaohsiung 80778, Taiwan \\ * Correspondence: tang@nkust.edu.tw (H.-C.T.); 1105407103@nkust.edu.tw (S.-T.Y.)
}

Received: 4 April 2019; Accepted: 10 May 2019; Published: 17 May 2019

\begin{abstract}
This paper analyzes the counterintuitive behaviors of adopted twelve distance-based similarity measures between intuitionistic fuzzy sets. Among these distance-based similarity measures, the largest number of components of the distance in the similarity measure is four. We propose six general counterintuitive test problems to analyze their counterintuitive behaviors. The results indicate that all the distance-based similarity measures have some counterintuitive test problems. Furthermore, for the largest number of components of the distance-based similarity measure, four types of counterintuitive examples exist. Therefore, the counterintuitive behaviors are inevitable for the distance-based similarity measures between intuitionistic fuzzy sets.
\end{abstract}

Keywords: intuitionistic fuzzy set; similarity; counterintuitive

\section{Introduction}

Fuzzy sets (FSs) theory, proposed by Zadeh [1], has successfully been applied in various fields. As a generalization of FSs, intuitionistic fuzzy sets (IFSs) proposed by Atanassov [2] is characterized by a membership function and a non-membership function. IFSs have been widely applied in cluster analysis [3-5], decision-making [6-11], medical diagnosis [12,13] and pattern recognition [14-19].

A similarity measure between two IFSs represents the alignment of the two sets. There are a large number of papers discussing various similarity measures between two IFSs [20-30]. A comprehensive and accurate survey of state-of-the-art research on IFSs and similarity measures are given by Atanassov [31] and Pedrycz and Chen [9]. The degree of similarity measure is an important tool for cluster analysis [3], decision-making [6,9,11], medical diagnosis [13] and pattern recognition [14-19,22]. One of the typical definitions of a similarity measure is the one-minus distance between two vectors [17,21-30]. The first distances over intuitionistic fuzzy sets are introduced by Atanassov [32]. Szmidt and Kacprzyk [33] adopted the normalized Hamming distance and the normalized Euclidean distance to present the similarity measures for IFSs. Furthermore, Szmidt and Kacprzyk [33] used hesitancy along with the membership and non-membership values of the elements in the sets being compared. The components of Chen's [21] similarity measure and Dengfeng and Chuntian's [23] similarity measure are concerned with the difference between degrees of support. The components of the Hong and Kim's [26] similarity measure, Mitchell's [17] similarity measure, the first Liang and Shi's [30] similarity measure, Hung and Yang's [27] three similarity measures and Li, Zhongxian and Degin's [29] similarity measure are the combination of the difference between membership degrees and the difference between non-membership degrees. The components of the Li and Xu's [24] similarity measure are the combination of the difference between membership degrees, the difference between non-membership degrees and the difference between degrees of support. The second Liang and Shi's [30] similarity measure is concerned with the combination of the difference between the first quartiles of intervals of membership degree and the difference between the third quartiles of intervals of membership degree. The third Liang and Shi's [31] similarity measure considers the combination of the 
difference between membership degrees, the difference between non-membership degrees, the difference between degrees of support and the difference between hesitant degrees. Therefore, the components of the distance can be the combination of the difference between membership degrees, the difference between non-membership degrees, the difference between hesitant degrees, the difference between degrees of support, the difference between the first quartiles of intervals of the membership degree and the difference between the third quartiles of intervals of the membership degree. Then we apply Minkowski distance to calculate the similarity degree between two IFSs. This paper focuses on these distance-based similarity measures.

In the literature, many varied approaches for the similarity measures between two IFSs are inconsistent with our intuition [14-30]. Two typical counterintuitive examples are (1) quite distinct IFSs having high similarity to each other and (2) two very distinct IFSs appearing to have equal similarity to a third IFS. However, these two typical counterintuitive ones are simple examples in the literature. This paper proposes six general counterintuitive test problems to analyze the counterintuitive behaviors of the distance-based similarity measures. As Li et al. [28] indicates, third Liang and Shi's [30] similarity measure has "... no counterintuitive cases". We further present four types of counterintuitive test problems for the third Liang and Shi's [30] similarity measure.

The main contribution of this paper is to propose six general counterintuitive test problems. To the best of our knowledge, the general test problems have not been yet presented to analyze the counterintuitive behaviors of the distance-based similarity measures. Moreover, the proposed general counterintuitive test problems can be used to analyze the counterintuitive behaviors of any similarity measures.

The organization of this paper is as follows. Section 2 briefly reviews the FSs and IFSs and presents the distance-based similarity measures. Section 3 presents six general counterintuitive test problems and analyzes the counterintuitive behaviors of distance-based similarity measures. Section 4 further presents the counterintuitive behaviors of the third Liang and Shi's [31] similarity measure. Finally, some concluding remarks and future research are presented.

\section{IFSs and Similarity Measures}

We firstly review the basic notations of FSs and IFSs. Let $X=\left\{x_{1}, x_{2}, \ldots, x_{n}\right\}$ be a non-empty universal set of real numbers $\mathcal{R}$.

Definition 1. An FS A over $X$ is defined by a membership function

$$
\mu_{A}: X \rightarrow[0,1] \text { for } 1 \leq i \leq n .
$$

Then the fuzzy set A over $X$ is the set

$$
A=\left\{\left(x_{i}, \mu_{A}\left(x_{i}\right)\right) \mid 1 \leq i \leq n\right\}
$$

We denote the set of all FSs over X by FS(X).

Definition 2. An IFS A over $\mathrm{X}$ is defined as

$$
A=\left\{\left(x_{i}, \mu_{A}\left(x_{i}\right), v_{A}\left(x_{i}\right)\right) \mid 1 \leq i \leq n\right\},
$$

where the membership function $\mu_{A}: X \rightarrow[0,1]$ and non-membership function $v_{A}: X \rightarrow[0,1]$ of the $x_{i}$ to the set A satisfy

$$
\mu_{A}\left(x_{i}\right)+v_{A}\left(x_{i}\right) \leq 1 .
$$

The set of all IFSs over $X$ is denoted by IFS(X). The degree of hesitancy associated with each $x_{i}$ is defined as

$$
\pi_{A}\left(x_{i}\right)=1-\mu_{A}\left(x_{i}\right)-v_{A}\left(x_{i}\right)
$$


measuring the lack of information or certitude.

We now briefly review some operations involving IFSs.

Definition 3. Let $A=\left\{\left(x_{i}, \mu_{A}\left(x_{i}\right), v_{A}\left(x_{i}\right)\right) \mid 1 \leq i \leq n\right\}$ and $B=\left\{\left(x_{i}, \mu_{B}\left(x_{i}\right), v_{B}\left(x_{i}\right)\right) \mid 1 \leq i \leq n\right\}$ be two IFSs. Then,

1. $A \subseteq B$ if and only if $\mu_{A}\left(x_{i}\right) \leq \mu_{B}\left(x_{i}\right)$ and $v_{A}\left(x_{i}\right) \geq v_{B}\left(x_{i}\right)$ for $1 \leq i \leq n$.

2. $A=B$ if and only if $\mu_{A}\left(x_{i}\right)=\mu_{B}\left(x_{i}\right)$ and $v_{A}\left(x_{i}\right)=v_{B}\left(x_{i}\right)$ for $1 \leq i \leq n$.

3. The complement of $A$ is defined as $A_{c}=\left\{\left(x_{i}, v_{A}\left(x_{i}\right), \mu_{A}\left(x_{i}\right)\right) \mid 1 \leq i \leq n\right\}$.

4. We denote the pure intuitionistic fuzzy set by $P I=\left\{\left(x_{i}, 0,0\right) \mid 1 \leq i \leq n\right\}$.

For the fuzzy set $A \in F S(X), \pi_{A}\left(x_{i}\right)=0$ for every $x_{i} \in X$, and for the pure intuitionistic fuzzy set, $\pi_{P I}\left(x_{i}\right)=1$ for every $x_{i} \in X$.

We now recall the definition of similarity measures between two IFSs.

Definition 4. A similarity measure $S: \operatorname{IFS}(X)^{2} \rightarrow[0,1]$ should satisfy the following properties:

1. $S(A, B) \in[0,1]$.

2. $S(A, B)=1$ if and only if $A=B$.

3. $S(A, B)=S(B, A)$.

4. If $A \subseteq B \subseteq C$, then $S(A, C) \leq S(A, B)$ and $S(A, C) \leq S(B, C)$.

So far, in the literature, many papers have been dedicated to problems connected with the similarity measures between two IFSs and research on this area is still carried on. Let $A=$ $\left\{\left(x_{i}, \mu_{A}\left(x_{i}\right), v_{A}\left(x_{i}\right)\right) \mid 1 \leq i \leq n\right\}$ and $B=\left\{\left(x_{i}, \mu_{B}\left(x_{i}\right), v_{B}\left(x_{i}\right)\right) \mid 1 \leq i \leq n\right\}$ be two IFSs. The existing distance-based similarity measures $S(A, B)$ between two IFSs $A$ and $B$ are defined as follows:

Chen [21]:

$$
S_{C}(A, B)=1-\frac{1}{2 n} \sum_{i=1}^{n}\left|\mu_{A}\left(x_{i}\right)-v_{A}\left(x_{i}\right)-\left(\mu_{B}\left(x_{i}\right)-v_{B}\left(x_{i}\right)\right)\right|
$$

Hong and Kim [26]:

$$
S_{H K}(A, B)=1-\frac{1}{2 n} \sum_{i=1}^{n}\left|\mu_{A}\left(x_{i}\right)-\mu_{B}\left(x_{i}\right)\right|+\left|v_{A}\left(x_{i}\right)-v_{B}\left(x_{i}\right)\right|
$$

$\mathrm{Li}$ and $\mathrm{Xu}[24]$ :

$$
\begin{aligned}
S_{L X}(A, B) & =1-\frac{1}{4 n} \sum_{i=1}^{n}\left\{\left|\mu_{A}\left(x_{i}\right)-\mu_{B}\left(x_{i}\right)\right|+\left|v_{A}\left(x_{i}\right)-v_{B}\left(x_{i}\right)\right|\right. \\
& \left.+\left|\mu_{A}\left(x_{i}\right)-v_{A}\left(x_{i}\right)-\left(\mu_{B}\left(x_{i}\right)-v_{B}\left(x_{i}\right)\right)\right|\right\}
\end{aligned}
$$

Dengfeng and Chuntian [23]:

$$
S_{D C}(A, B)=1-\sqrt[p]{\frac{1}{2 n} \sum_{i=1}^{n}\left|\mu_{A}\left(x_{i}\right)-v_{A}\left(x_{i}\right)-\left(\mu_{B}\left(x_{i}\right)-v_{B}\left(x_{i}\right)\right)\right|^{p}}
$$

Mitchell [17]:

$$
S_{M}(A, B)=1-\frac{1}{2}\left\{\sqrt[p]{\frac{1}{n} \sum_{i=1}^{n}\left|\mu_{A}\left(x_{i}\right)-\mu_{B}\left(x_{i}\right)\right|^{p}}+\sqrt[p]{\frac{1}{n} \sum_{i=1}^{n}\left|v_{A}\left(x_{i}\right)-v_{B}\left(x_{i}\right)\right|^{p}}\right\}
$$


Liang and Shi [30]:

$$
\begin{gathered}
S_{L S 1}(A, B)=1-\frac{1}{2} \sqrt[p]{\frac{1}{n} \sum_{i=1}^{n}\left(\left|\mu_{A}\left(x_{i}\right)-\mu_{B}\left(x_{i}\right)\right|+\left|v_{A}\left(x_{i}\right)-v_{B}\left(x_{i}\right)\right|\right)^{p}} \\
S_{L S 2}(A, B)=1-\frac{1}{8}\left(\frac { 1 } { n } \sum _ { i = 1 } ^ { n } \left(\left|3\left(\mu_{A}\left(x_{i}\right)-\mu_{B}\left(x_{i}\right)\right)-\left(v_{A}\left(x_{i}\right)-v_{B}\left(x_{i}\right)\right)\right|+\mid\left(\mu_{A}\left(x_{i}\right)-\right.\right.\right. \\
\left.\left.\left.\mu_{B}\left(x_{i}\right)\right)-3\left(v_{A}\left(x_{i}\right)-v_{B}\left(x_{i}\right)\right) \mid\right)^{p}\right)^{1 / p} \\
S_{L S 3}(A, B)=1-\frac{1}{2}\left(\frac { 1 } { 3 n } \sum _ { i = 1 } ^ { n } \left(\left|\mu_{A}\left(x_{i}\right)-\mu_{B}\left(x_{i}\right)\right|+\left|v_{A}\left(x_{i}\right)-v_{B}\left(x_{i}\right)\right|+\mid \mu_{A}\left(x_{i}\right)-v_{A}\left(x_{i}\right)-\right.\right. \\
\left.\left.\left(\mu_{B}\left(x_{i}\right)-v_{B}\left(x_{i}\right)\right)|+| \mu_{A}\left(x_{i}\right)+v_{A}\left(x_{i}\right)-\left(\mu_{B}\left(x_{i}\right)+v_{B}\left(x_{i}\right)\right) \mid\right)^{p}\right)^{1 / p}
\end{gathered}
$$

Hung and Yang [27]:

$$
\begin{gathered}
S_{H Y 1}(A, B)=1-\frac{1}{n} \sum_{i=1}^{n} \max \left\{\left|\mu_{A}\left(x_{i}\right)-\mu_{B}\left(x_{i}\right)\right|,\left|v_{A}\left(x_{i}\right)-v_{B}\left(x_{i}\right)\right|\right\} \\
S_{H Y 2}(A, B)=\frac{e^{-\frac{1}{n} \sum_{i=1}^{n} \max \left\{\left|\mu_{A}\left(x_{i}\right)-\mu_{B}\left(x_{i}\right)\right|,\left|v_{A}\left(x_{i}\right)-v_{B}\left(x_{i}\right)\right|\right\}}-e^{-1}}{1-e^{-1}} \\
S_{H Y 3}(A, B)=\frac{1-\frac{1}{n} \sum_{i=1}^{n} \max \left\{\left|\mu_{A}\left(x_{i}\right)-\mu_{B}\left(x_{i}\right)\right|,\left|v_{A}\left(x_{i}\right)-v_{B}\left(x_{i}\right)\right|\right\}}{1+\frac{1}{n} \sum_{i=1}^{n} \max \left\{\left|\mu_{A}\left(x_{i}\right)-\mu_{B}\left(x_{i}\right)\right|,\left|v_{A}\left(x_{i}\right)-v_{B}\left(x_{i}\right)\right|\right\}}
\end{gathered}
$$

Li, Zhongxian and Degin [29]:

$$
S_{L Z D}(A, B)=1-\sqrt{\frac{1}{2 n} \sum_{i=1}^{n}\left(\mu_{A}\left(x_{i}\right)-\mu_{B}\left(x_{i}\right)\right)^{2}+\left(v_{A}\left(x_{i}\right)-v_{B}\left(x_{i}\right)\right)^{2}} .
$$

Therefore, we adopt twelve distance-based similarity measures. When $p=1$, we have

$$
\begin{gathered}
S_{D C}(A, B)=S_{C}(A, B) \\
S_{M}(A, B)=1-\frac{1}{2 n} \sum_{i=1}^{n}\left|\mu_{A}\left(x_{i}\right)-\mu_{B}\left(x_{i}\right)\right|+\left|v_{A}\left(x_{i}\right)-v_{B}\left(x_{i}\right)\right|=S_{H K}(A, B)
\end{gathered}
$$

and

$$
S_{L S 1}(A, B)=1-\frac{1}{2 n} \sum_{i=1}^{n}\left|\mu_{A}\left(x_{i}\right)-\mu_{B}\left(x_{i}\right)\right|+\left|v_{A}\left(x_{i}\right)-v_{B}\left(x_{i}\right)\right|=S_{H K}(A, B) .
$$

Among these distance-based similarity measures, four types of components of distance are distinguished: (I) the combination of difference between membership degrees and difference between non-membership degrees; (II) the difference between hesitant degrees; (III) the difference between degrees of support and (IV) the combination of difference between the first quartiles of intervals of membership degree and the difference between the third quartiles of intervals of membership degree. Detailed results are presented in Table 1 . The similarity measures $S_{H K}(A, B), S_{M}(A, B), S_{L S 1}(A, B)$, $S_{H Y 1}(A, B), S_{H Y 2}(A, B), S_{H Y 3}(A, B)$ and $S_{L Z D}(A, B)$ are dependent on type I. The similarity measures $S_{C}(A, B)$ and $S_{D C}(A, B)$ are concerned with type III. $S_{L X}(A, B)$ is dependent on the combination of types I and III. $S_{L S 2}(A, B)$ is concerned with type IV. $S_{L S 3}(A, B)$ is concerned with the combination of types I, II and III. The largest number of components of the distance in the similarity measure is four for the similarity measure $S_{L S 3}(A, B)$. Therefore, we anticipate that the intuitive behavior of $S_{L S 3}(A, B)$ is superior to those of other similarity measures. 
Table 1. The four types of components of distance-based similarity measures.

\begin{tabular}{lllll}
\hline Similarity & I & II & III & IV \\
\hline$S_{C}(A, B)$ & & & $\sqrt{ }$ & \\
$S_{H K}(A, B)$ & $\sqrt{ }$ & & & \\
$S_{L X}(A, B)$ & $\sqrt{ }$ & & $\sqrt{ }$ & \\
$S_{D C}(A, B)$ & & & $\sqrt{ }$ & \\
$S_{M}(A, B)$ & $\sqrt{ }$ & & & \\
$S_{L S 1}(A, B)$ & $\sqrt{ }$ & & & $\sqrt{ }$ \\
$S_{L S 2}(A, B)$ & & & & \\
$S_{L S 3}(A, B)$ & $\sqrt{ }$ & $\sqrt{ }$ & & \\
$S_{H Y 1}(A, B)$ & $\sqrt{ }$ & & & \\
$S_{H Y 2}(A, B)$ & $\sqrt{ }$ & & & \\
$S_{H Y 3}(A, B)$ & $\sqrt{ }$ & & & \\
$S_{L Z D}(A, B)$ & $\sqrt{ }$ & & \\
\hline
\end{tabular}

\section{General Counterintuitive Test Problems}

Much literature has been written on the counterintuitive examples for the similarity measures between two IFSs. Two typical counterintuitive examples are (I) $S(A, B)=1$ for $A \neq B, A, B \in \operatorname{IFS}(X)$ and (II) $S\left(P_{1}, Q\right)=S\left(P_{2}, Q\right)$ for $P_{1} \neq P_{2}, P_{1}, P_{2}, Q \in \operatorname{IFS}(X)$. This paper proposes six general counterintuitive test problems presented in Table 2. From Definition 4 , a similarity measure which fails type I is not a similarity measure.

Table 2. The six general counterintuitive test problems.

\begin{tabular}{cr}
\hline$\#$ & Test Problem \\
\hline T1 & $A(a, a), B(b, b), a \neq b, 0 \leq a, b \leq 1 / 2$ satisfying $S(A, B)=1$. \\
T2 & $A(a, a), B(b, b), C(a, b), D(b, a), a \neq b, 0 \leq a, b \leq 1 / 2$ satisfying $S(A, B)=S(C, D)$. \\
T3 & $P_{1}(a, b), P_{2}\left(\frac{a+b}{2}, \frac{a+b}{2}\right), Q(b, b), a \neq b, 0 \leq a, b, a+b \leq 1, b \leq 1 / 2$ satisfying $S\left(P_{1}, Q\right)=S\left(P_{2}, Q\right)$. \\
T4 & $P_{1}(1,0), P_{2}(a, 1-a), Q(0,0), 0 \leq a \leq 1$ satisfying $S\left(P_{1}, Q\right)=S\left(P_{2}, Q\right)$. \\
T5 & $P_{1}(b, b-\alpha), P_{2}(b, a-\alpha), Q(a, a-\alpha), a \neq b, \alpha \leq a, b, a+b-\alpha \leq 1,2 a-\alpha \leq 1,2 b-\alpha \leq 1$ \\
T6 & $P_{1}\{(a+\alpha, b),(a, b+\alpha)\}, P_{2}\{(a+\alpha, b+\alpha),(a+\alpha, b+\alpha)\}, Q\{(a, b),(a+\alpha, b)\}, a \neq b, 0 \leq$ \\
& $a, b, \alpha, a+b+2 \alpha \leq 1$ satisfying $S\left(P_{1}, Q\right)=S\left(P_{2}, Q\right)$. \\
\hline
\end{tabular}

Test problem $\mathrm{T} 1$ is a type I counterintuitive one. So, a similarity measure which fails test problem T1 is not a similarity measure. Test problem T2 is to distinguish the positive difference and negative difference. Test problems T3, T4, T5 and T6 can be considered pattern recognition problems. Consider two known patterns $P_{1}$ and $P_{2}$. We want to classify an unknown pattern represented by $Q$ into one of the patterns $P_{1}$ and $P_{2}$. For test problem T3, the membership degree and non-membership degree of $P_{2}$ is the average of those of $P_{1}$ and $Q$. For $S\left(P_{1}, Q\right)=S\left(P_{2}, Q\right)$, it implies that T3 is a counterintuitive test problem. For test problem T4 with $S\left(P_{1}, Q\right)=S\left(P_{2}, Q\right)$, a crisp set $P_{1}$ is as similar to PI $Q$ as any FS $P_{2}$. So T4 is a counterintuitive test problem. For test problem T5, although

$$
\left|\mu_{P_{1}}-\mu_{Q}\right| \neq\left|\mu_{P_{2}}-\mu_{Q}\right| \text { or }\left|v_{P_{1}}-v_{Q}\right| \neq \mid v_{P_{2}}-v_{Q}
$$

and

$$
\left|\mu_{P_{1}}-\mu_{Q}\right|+\left|v_{P_{1}}-v_{Q}\right| \neq\left|\mu_{P_{2}}-\mu_{Q}\right|+\left|v_{P_{2}}-v_{Q}\right|
$$

but

$$
\left|\mu_{P_{1}}-\mu_{Q}\right|+\left|v_{P_{1}}-v_{Q}\right|+\left|\mu_{P_{1}}-v_{P_{1}}-\left(\mu_{Q}-v_{Q}\right)\right|=\left|\mu_{P_{2}}-\mu_{Q}\right|+\left|v_{P_{2}}-v_{Q}\right|+\left|\mu_{P_{2}}-v_{P_{2}}-\left(\mu_{Q}-v_{Q}\right)\right|,
$$


then $S\left(P_{1}, Q\right)=S\left(P_{2}, Q\right)$. It follows that $\mathrm{T} 5$ is a counterintuitive test problem. For test problem $\mathrm{T} 6$, if

$$
\begin{aligned}
\sum_{i=1}^{2}\left(\left|\mu_{P_{1}}\left(x_{i}\right)-\mu_{Q}\left(x_{i}\right)\right|+\left|v_{P_{1}}\left(x_{i}\right)-v_{Q}\left(x_{i}\right)\right|+\left|\mu_{P_{1}}\left(x_{i}\right)-v_{P_{1}}\left(x_{i}\right)-\left(\mu_{Q}\left(x_{i}\right)-v_{Q}\left(x_{i}\right)\right)\right|\right. & \\
& \left.+\left|\mu_{P_{1}}\left(x_{i}\right)+v_{P_{1}}\left(x_{i}\right)-\left(\mu_{Q}\left(x_{i}\right)+v_{Q}\left(x_{i}\right)\right)\right|\right) \\
& =\sum_{i=1}^{2}\left(\left|\mu_{P_{2}}\left(x_{i}\right)-\mu_{Q}\left(x_{i}\right)\right|+\left|v_{P_{2}}\left(x_{i}\right)-v_{Q}\left(x_{i}\right)\right|+\mid \mu_{P_{2}}\left(x_{i}\right)-v_{P_{2}}\left(x_{i}\right)\right. \\
& \left.-\left(\mu_{Q}\left(x_{i}\right)-v_{Q}\left(x_{i}\right)\right)|+| \mu_{P_{2}}\left(x_{i}\right)+v_{P_{2}}\left(x_{i}\right)-\left(\mu_{Q}\left(x_{i}\right)+v_{Q}\left(x_{i}\right)\right) \mid\right)
\end{aligned}
$$

then $S\left(P_{1}, Q\right)=S\left(P_{2}, Q\right)$. So T6 is a counterintuitive test problem.

For each distance-based similarity measure, we analyze the counterintuitive behaviors of the six general counterintuitive test problems as follows.

For similarity measure $S_{C}(A, B)$, since $S_{C}(A, B)=1$ for test problem T1, it implies that T1 is the counterintuitive test problem for the similarity measure $S_{C}(A, B)$.

For similarity measure $S_{H K}(A, B)$, we have

$$
\begin{gathered}
S_{H K}(A, B)=S_{H K}(C, D)=1-|a-b| \\
S_{H K}\left(P_{1}, Q\right)=S_{H K}\left(P_{2}, Q\right)=1-\frac{|a-b|}{2}
\end{gathered}
$$

and

$$
S_{H K}\left(P_{1}, Q\right)=S_{H K}\left(P_{2}, Q\right)=0.5
$$

for test problems T2, T3 and T4, respectively. So the counterintuitive test problems for the similarity measure $S_{H K}(A, B)$ are $\mathrm{T} 2, \mathrm{~T} 3$ and $\mathrm{T} 4$.

The counterintuitive test problem for the similarity measure $S_{L X}(A, B)$ is $\mathrm{T} 5$, because

$$
S_{L X}\left(P_{1}, Q\right)=S_{L X}\left(P_{2}, Q\right)=1-\frac{|a-b|}{2}
$$

A similar argument shows that the counterintuitive test problems for the similarity measures $S_{D C}(A, B)$ and $S_{M}(A, B)$ are test problem $\mathrm{T} 1$ and test problems $\mathrm{T} 2, \mathrm{~T} 3, \mathrm{~T} 4$, respectively. The counterintuitive test problems for the three Liang and Shi's similarity measures $S_{L S 1}(A, B)$, $S_{L S 2}(A, B)$ and $S_{L S 3}(A, B)$ are test problems $\mathrm{T} 2, \mathrm{~T} 3, \mathrm{~T} 4$, test problem $\mathrm{T} 5$, and test problem $\mathrm{T} 6$, respectively. The counterintuitive test problems are T2 and T5 for the three Hung and Yang's similarity measures $S_{H Y 1}(A, B), S_{H Y 2}(A, B)$ and $S_{H Y 3}(A, B)$, and T2 for $S_{L Z D}(A, B)$. Detailed results are displayed in Table 3. $S_{C}(A, B)$ and $S_{D C}(A, B)$ are not similarity measures. The smallest number of counterintuitive test problems is one. These counterintuitive test problems are T2 for $S_{L Z D}(A, B)$, T5 for $S_{L X}(A, B), S_{L S 2}(A, B)$, and T6 for $S_{L S 3}(A, B)$. Therefore, the counterintuitive test problem is $\mathrm{T} 1$ for $S_{C}(A, B)$ and $S_{D C}(A, B)$, T2 for $S_{H K}(A, B), S_{M}(A, B), S_{L S 1}(A, B), S_{H Y 1}(A, B), S_{H Y 2}(A, B), S_{H Y 3}(A, B)$ and $S_{L Z D}(A, B)$, T3 and T4 for $S_{H K}(A, B), S_{M}(A, B)$ and $S_{L S 1}(A, B)$, T5 for $S_{L X}(A, B), S_{L S 2}(A, B)$, $S_{H Y 1}(A, B), S_{H Y 2}(A, B)$ and $S_{H Y 3}(A, B)$, and T6 for $S_{L S 3}(A, B)$. 
Table 3. The counterintuitive test problems of distance-based similarity measures.

\begin{tabular}{cc}
\hline Similarity & Counterintuitive Test Problems \\
\hline$S_{C}(\boldsymbol{A}, \boldsymbol{B})$ & $\mathrm{T} 1$ \\
$S_{H K}(\boldsymbol{A}, \boldsymbol{B})$ & $\mathrm{T} 2, \mathrm{~T} 3, \mathrm{~T} 4$ \\
$S_{L X}(\boldsymbol{A}, \boldsymbol{B})$ & $\mathrm{T} 5$ \\
$S_{D C}(\boldsymbol{A}, \boldsymbol{B})$ & $\mathrm{T} 1$ \\
$S_{M}(\boldsymbol{A}, \boldsymbol{B})$ & $\mathrm{T} 2, \mathrm{~T} 3, \mathrm{~T} 4$ \\
$S_{L S 1}(\boldsymbol{A}, \boldsymbol{B})$ & $\mathrm{T} 2, \mathrm{~T} 3, \mathrm{~T} 4$ \\
$S_{L S 2}(\boldsymbol{A}, \boldsymbol{B})$ & $\mathrm{T} 5$ \\
$S_{L S 3}(\boldsymbol{A}, \boldsymbol{B})$ & $\mathrm{T} 6$ \\
$S_{H Y 1}(\boldsymbol{A}, \boldsymbol{B})$ & $\mathrm{T} 2, \mathrm{~T} 5$ \\
$S_{H Y 2}(\boldsymbol{A}, \boldsymbol{B})$ & $\mathrm{T} 2, \mathrm{~T} 5$ \\
$S_{H Y 3}(\boldsymbol{A}, \boldsymbol{B})$ & $\mathrm{T} 2, \mathrm{~T} 5$ \\
$S_{L Z D}(\boldsymbol{A}, \boldsymbol{B})$ & $\mathrm{T} 2$ \\
\hline
\end{tabular}

\section{Counterintuitive Test Problem for $S_{L S 3}(A, B)$}

From the analysis of counterintuitive test problems in the previous section and those of Li et al. [28], the third Liang and Shi's similarity measure $S_{L S 3}(A, B)$ outperforms other similarity measures. This section further analyzes the counterintuitive behaviors of the third Liang and Shi's similarity measure $S_{L S 3}(A, B)$.

Consider the four types of three IFSs $A, B$ and $C$ as follows.

(I) $A\left(a_{1}, a_{2}\right), B\left(a_{1}, a_{2}+\alpha\right)$ and $C\left(c_{1}, a_{2}+\alpha-\beta\right)$ for $c_{1} \geq a_{1}$.

(II) $A\left(a_{1}, a_{2}\right), B\left(a_{1}, a_{2}+\alpha\right)$ and $C\left(c_{1}, a_{2}+\alpha-\beta\right)$ for $a_{1} \geq c_{1}$.

(III) $A\left(a_{1}, a_{2}\right), B\left(a_{1}, a_{2}-\alpha\right)$ and $C\left(c_{1}, a_{2}-\alpha+\beta\right)$ for $c_{1} \geq a_{1}$.

(IV) $A\left(a_{1}, a_{2}\right), B\left(a_{1}, a_{2}-\alpha\right)$ and $C\left(c_{1}, a_{2}-\alpha+\beta\right)$ for $a_{1} \geq c_{1}$.

By fixing $A\left(a_{1}, a_{2}\right)$ and $B\left(a_{1}, a_{2} \pm \alpha\right)$, we will present the explicit form of IFS $C\left(c_{1}, a_{2} \pm \alpha \mp \beta\right)$ satisfying $S_{L S 3}(A, B)=S_{L S 3}(A, C)$. This implies that $\left\{A\left(a_{1}, a_{2}\right), B\left(a_{1}, a_{2} \pm \alpha\right), C\left(c_{1}, a_{2} \pm \alpha \mp \beta\right)\right\}$ is a counterintuitive example for the similarity measure $S_{L S 3}(A, B)$.

We now analyze type I. From $A\left(a_{1}, a_{2}\right), B\left(a_{1}, a_{2}+\alpha\right), C\left(c_{1}, a_{2}+\alpha-\beta\right)$, and $c_{1} \geq a_{1}$, it follows that

$$
S_{L S 3}(A, B)=3 \alpha
$$

and

$$
S_{L S 3}(A, C)=c_{1}-a_{1}+|\alpha-\beta|+\left|c_{1}-a_{1}-(\alpha-\beta)\right|+\left|c_{1}-a_{1}+\alpha-\beta\right|
$$

Four cases are considered as follows.

Case I1: $\alpha-\beta \geq 0, c_{1}-a_{1}-(\alpha-\beta) \leq 0$ and $c_{1}-a_{1}+\alpha-\beta \geq 0$.

We get that

$$
S_{L S 3}(A, C)=-a_{1}+c_{1}+3 \alpha-3 \beta .
$$

In order to $S_{L S 3}(A, B)=S_{L S 3}(A, C)$, we have that $c_{1}=a_{1}+3 \beta$, implying

$$
C\left(a_{1}+3 \beta, a_{2}+\alpha-\beta\right) .
$$

The following conditions should be satisfied for $A, B, C \in \operatorname{IFS}(X)$ and $c_{1} \geq a_{1}$,

$$
a_{1}-c_{1}+\alpha-\beta=\alpha-4 \beta \geq 0, a_{1}+a_{2}+\alpha \leq 1, a_{1}+a_{2}+\alpha+2 \beta \leq 1, a_{1}, a_{2}, \alpha, \beta \geq 0 .
$$

Therefore, if $\alpha \geq 4 \beta, a_{1}+a_{2}+\alpha+2 \beta \leq 1, a_{1}, a_{2}, \alpha, \beta \geq 0$, then $A\left(a_{1}, a_{2}\right), B\left(a_{1}, a_{2}+\alpha\right)$ and $C\left(a_{1}+3 \beta, a_{2}+\alpha-\beta\right)$ satisfying $S_{L S 3}(A, B)=S_{L S 3}(A, C)$.

A similar argument shows the other three cases as follows. Detailed results of $C\left(c_{1}, a_{2}+\alpha-\beta\right)$ and conditions for $c_{1} \geq a_{1}$ are summarized in Table 4 .

Case I2: $\alpha-\beta \geq 0, c_{1}-a_{1}-(\alpha-\beta) \geq 0$ and $c_{1}-a_{1}+\alpha-\beta \geq 0$. 
If $\beta \leq \alpha \leq 4 \beta, a_{1}+a_{2}+\frac{5 \alpha-2 \beta}{3} \leq 1$ and $a_{1}, a_{2}, \alpha, \beta \geq 0$, then $A\left(a_{1}, a_{2}\right), B\left(a_{1}, a_{2}+\alpha\right)$ and $C\left(a_{1}+\frac{2 \alpha+\beta}{3}, a_{2}+\alpha-\beta\right)$ satisfying $S_{L S 3}(A, B)=S_{L S 3}(A, C)$.

Case I3: $\alpha-\beta \leq 0, c_{1}-a_{1}-(\alpha-\beta) \geq 0$ and $c_{1}-a_{1}+\alpha-\beta \geq 0$.

If $4 \beta / 7 \leq \alpha \leq \beta, a_{1}+a_{2}+\alpha \leq 1$ and $a_{1}, a_{2}, \alpha, \beta \geq 0$, then $A\left(a_{1}, a_{2}\right), B\left(a_{1}, a_{2}+\alpha\right)$ and $C\left(a_{1}+\frac{4 \alpha-\beta}{3}, a_{2}+\alpha-\beta\right)$ satisfying $S_{L S 3}(A, B)=S_{L S 3}(A, C)$.

Case I4: $\alpha-\beta \leq 0, c_{1}-a_{1}-(\alpha-\beta) \geq 0$ and $c_{1}-a_{1}+\alpha-\beta \leq 0$.

If $\beta / 2 \leq \alpha \leq 4 \beta / 7, a_{1}+a_{2}+\alpha \leq 1$ and $a_{1}, a_{2}, \alpha, \beta \geq 0$, then $A\left(a_{1}, a_{2}\right), B\left(a_{1}, a_{2}+\alpha\right)$ and $C\left(a_{1}+6 \alpha-3 \beta, a_{2}+\alpha-\beta\right)$ satisfying $S_{L S 3}(A, B)=S_{L S 3}(A, C)$.

Table 4. $A\left(a_{1}, a_{2}\right), B\left(a_{1}, a_{2}+\alpha\right)$ and $C$ satisfying $S_{L S 3}(A, B)=S_{L S 3}(A, C), a_{1}, a_{2}, \alpha, \beta \geq 0$.

\begin{tabular}{ccc}
\hline & $C\left(c_{1}, a_{2}+\alpha-\beta\right)$ & Conditions \\
\hline I1 & $C\left(a_{1}+3 \beta, a_{2}+\alpha-\beta\right)$ & $c_{1} \geq a_{1}, \alpha \geq 4 \beta, a_{1}+a_{2}+\alpha+2 \beta \leq 1$ \\
I2 & $C\left(a_{1}+\frac{2 \alpha+\beta}{3}, a_{2}+\alpha-\beta\right)$ & $c_{1} \geq a_{1}, \beta \leq \alpha \leq 4 \beta, a_{1}+a_{2}+\frac{5 \alpha-2 \beta}{3} \leq 1$ \\
I3 & $C\left(a_{1}+\frac{4 \alpha-\beta}{3}, a_{2}+\alpha-\beta\right)$ & $c_{1} \geq a_{1}, 4 / 7 \beta \leq \alpha \leq \beta, a_{1}+a_{2}+\alpha \leq 1$ \\
I4 & $C\left(a_{1}+6 \alpha-3 \beta, a_{2}+\alpha-\beta\right)$ & $c_{1} \geq a_{1}, 0.5 \beta \leq \alpha \leq 4 / 7 \beta, a_{1}+a_{2}+\alpha \leq 1$ \\
II1 & $C\left(a_{1}-3 \beta, a_{2}+\alpha-\beta\right)$ & $a_{1} \geq c_{1}, \alpha \geq 4 \beta, a_{1}+a_{2}+\alpha \leq 1$ \\
II2 & $C\left(a_{1}-\frac{2 \alpha+\beta}{3}, a_{2}+\alpha-\beta\right)$ & $a_{1} \geq c_{1}, \beta \leq \alpha \leq 4 \beta, a_{1}+a_{2}+\alpha \leq 1$ \\
II3 & $C\left(a_{1}-\frac{4 \alpha-\beta}{3}, a_{2}+\alpha-\beta\right)$ & $a_{1} \geq c_{1}, 4 / 7 \beta \leq \alpha \leq \beta, a_{1}+a_{2}+\alpha \leq 1$ \\
II4 & $C\left(a_{1}-6 \alpha+3 \beta, a_{2}+\alpha-\beta\right)$ & $a_{1} \geq c_{1}, 0.5 \beta \leq \alpha \leq 4 / 7 \beta, a_{1}+a_{2}+\alpha \leq 1$ \\
\hline
\end{tabular}

For type II, from $A\left(a_{1}, a_{2}\right), B\left(a_{1}, a_{2}+\alpha\right), C\left(c_{1}, a_{2}+\alpha-\beta\right)$ and $a_{1} \geq c_{1}$, it follows that Equation (10) and

$$
S_{L S 3}(A, C)=a_{1}-c_{1}+|\alpha-\beta|+\left|a_{1}-c_{1}+\alpha-\beta\right|+\left|a_{1}-c_{1}-\alpha+\beta\right|
$$

Four cases are considered as follows.

Case II1: $\alpha-\beta \geq 0, a_{1}-c_{1}+\alpha-\beta \geq 0$ and $a_{1}-c_{1}-\alpha+\beta \leq 0$.

Case II2: $\alpha-\beta \geq 0, a_{1}-c_{1}+\alpha-\beta \geq 0$ and $a_{1}-c_{1}-\alpha+\beta \geq 0$.

Case II3: $\alpha-\beta \leq 0, a_{1}-c_{1}+\alpha-\beta \geq 0$ and $a_{1}-c_{1}-\alpha+\beta \geq 0$.

Case II4: $\alpha-\beta \leq 0, a_{1}-c_{1}+\alpha-\beta \leq 0$ and $a_{1}-c_{1}-\alpha+\beta \geq 0$.

A similar argument presents the explicit form of $C\left(c_{1}, a_{2}+\alpha-\beta\right)$ and conditions for $a_{1} \geq c_{1}$ shown in Table 4. Given $A\left(a_{1}, a_{2}\right)$ and $B\left(a_{1}, a_{2}+\alpha\right)$, from $c_{1} \geq a_{1}$ of type I to $a_{1} \geq c_{1}$ of type II, the form of $C$ from $C\left(a_{1}+\gamma_{i}, a_{2}+\alpha-\beta\right)$ of type I case Ii to $C\left(a_{1}-\gamma_{i}, a_{2}+\alpha-\beta\right)$ of type II case IIi, for each case, where $\gamma_{i}$ is the $i$ th component of $\left[3 \beta, \frac{2 \alpha+\beta}{3}, \frac{4 \alpha-\beta}{3}, 6 \alpha-3 \beta\right]$.

By symmetric argument, the form of $C\left(c_{1}, a_{2}-\alpha+\beta\right)$ and conditions for types III and IV are presented in Table 5. From Table 5, given $A\left(a_{1}, a_{2}\right)$ and $B\left(a_{1}, a_{2}-\alpha\right)$, from $c_{1} \geq a_{1}$ of type III to $a_{1} \geq c_{1}$ of type IV, the form of $C$ from $C\left(a_{1}+\gamma_{i}, a_{2}-\alpha+\beta\right)$ of type III case IIIi to $C\left(a_{1}-\gamma_{i}, a_{2}-\alpha+\beta\right)$ of type IV case IVi, for each case, where $\gamma_{i}$ is the $i$ th component of $\left[3 \beta, \frac{2 \alpha+\beta}{3}, \frac{4 \alpha-\beta}{3}, 6 \alpha-3 \beta\right]$.

Table 5. $A\left(a_{1}, a_{2}\right), B\left(a_{1}, a_{2}-\alpha\right)$ and C satisfying $S_{L S 3}(A, B)=S_{L S 3}(A, C), a_{1}, a_{2}, \alpha, \beta \geq 0$.

\begin{tabular}{ccc}
\hline & $C\left(\mathbf{c}_{1}, a_{2}-\alpha+\beta\right)$ & Conditions \\
\hline III1 & $C\left(a_{1}+3 \beta, a_{2}-\alpha+\beta\right)$ & $c_{1} \geq a_{1}, \alpha \geq 4 \beta, a_{1}+a_{2}-\alpha+4 \beta \leq 1$ \\
III2 & $C\left(a_{1}+\frac{2 \alpha+\beta}{3}, a_{2}-\alpha+\beta\right)$ & $c_{1} \geq a_{1}, \beta \leq \alpha \leq 4 \beta, a_{1}+a_{2}+\frac{-\alpha+4 \beta}{3} \leq 1$ \\
III3 & $C\left(a_{1}+\frac{4 \alpha-\beta}{3}, a_{2}-\alpha+\beta\right)$ & $c_{1} \geq a_{1}, 4 / 7 \beta \leq \alpha \leq \beta, a_{1}+a_{2}+\frac{\alpha+2 \beta}{3} \leq 1$ \\
III4 & $C\left(a_{1}+6 \alpha-3 \beta, a_{2}-\alpha+\beta\right)$ & $c_{1} \geq a_{1}, 0.5 \beta \leq \alpha \leq 4 / 7 \beta, a_{1}+a_{2}+5 \alpha-2 \beta \leq 1$ \\
IV1 & $C\left(a_{1}-3 \beta, a_{2}-\alpha+\beta\right)$ & $a_{1} \geq c_{1}, \alpha \geq 4 \beta, a_{1}+a_{2}-\alpha \leq 1$ \\
IV2 & $C\left(a_{1}-\frac{2 \alpha+\beta}{3}, a_{2}-\alpha+\beta\right)$ & $a_{1} \geq c_{1}, \beta \leq \alpha \leq 4 \beta, a_{1}+a_{2}-\alpha \leq 1$ \\
IV3 & $C\left(a_{1}-\frac{4 \alpha-\beta}{3}, a_{2}-\alpha+\beta\right)$ & $a_{1} \geq c_{1}, 4 / 7 \beta \leq \alpha \leq \beta, a_{1}+a_{2}-\frac{7 \alpha-4 \beta}{3} \leq 1$ \\
IV4 & $C\left(a_{1}-6 \alpha+3 \beta, a_{2}-\alpha+\beta\right)$ & $a_{1} \geq c_{1}, 0.5 \beta \leq \alpha \leq 4 / 7 \beta, a_{1}+a_{2}-7 \alpha+4 \beta \leq 1$ \\
\hline
\end{tabular}


From Tables 4 and 5, we observe that there are 1-1 correspondences between the explicit forms of $C$ of four types. More precisely, for each case $i, C\left(a_{1}+\gamma_{i}, a_{2}+\alpha-\beta\right)$ of type I case Ii corresponds to $C\left(a_{1}-\gamma_{i}, a_{2}+\alpha-\beta\right)$ of type II case IIi, which, in turn, to $C\left(a_{1}+\gamma_{i}, a_{2}-\alpha+\beta\right)$ of type III case IIIi, which in turn to $C\left(a_{1}-\gamma_{i}, a_{2}-\alpha+\beta\right)$ of type IV case IVi, where $\gamma_{i}$ is the $i$ th component of $\left[3 \beta, \frac{2 \alpha+\beta}{3}, \frac{4 \alpha-\beta}{3}, 6 \alpha-3 \beta\right]$.

\section{Conclusions and Future Research}

This paper considers twelve distance-based similarity measures. Among which the largest number of components of the distance in the similarity measure is four for $S_{L S 3}(A, B)$. Therefore, we anticipate that the intuitive behaviors of $S_{L S 3}(A, B)$ is superior to those of other similarity measures. Much literature has been written on the counterintuitive examples for the similarity measures between two IFSs. This paper proposes six general counterintuitive test problems T1-T6. The counterintuitive test problem is T1 for $S_{C}(A, B)$ and $S_{D C}(A, B)$, T2 for $S_{H K}(A, B), S_{M}(A, B), S_{L S 1}(A, B), S_{H Y 1}(A, B)$, $S_{H Y 2}(A, B), S_{H Y 3}(A, B)$ and $S_{L Z D}(A, B)$, T3 and T4 for $S_{H K}(A, B), S_{M}(A, B)$ and $S_{L S 1}(A, B)$, T5 for $S_{L X}(A, B), S_{L S 2}(A, B), S_{H Y 1}(A, B), S_{H Y 2}(A, B)$ and $S_{H Y 3}(A, B)$, and T6 for $S_{L S 3}(A, B)$. For $S_{L S 3}(A, B)$, four types of counterintuitive example $\left\{A\left(a_{1}, a_{2}\right), B\left(a_{1}, a_{2} \pm \alpha\right), C\left(c_{1}, a_{2} \pm \alpha \mp \beta\right)\right\}$ exist. There are 1-1 correspondences between the explicit forms of $C$ of four types.

Worthy of future research is when the counterintuitive analysis is extended to other types of similarity measures. In particular, the analysis can be extended to the similarity measures based on the transformation techniques or based on the centroid points of transformation techniques. Thus, the counterintuitive analyses of the transformation techniques based or centroid points based similarity measures is a subject of considerable ongoing research.

Author Contributions: H.-C.T. analyzed the method and wrote the paper. S.-T.Y. performed the experiments.

Funding: This research received no external funding.

Conflicts of Interest: The authors declare that they have no competing interests.

\section{References}

1. Zadeh, L.A. Fuzzy sets. Inf. Control. 1965, 8, 338-356. [CrossRef]

2. Atanassov, K.T. Intuitionistic fuzzy sets. Fuzzy Sets Syst. 1986, 20, 87-96. [CrossRef]

3. Hwang, C.M.; Yang, M.S.; Hung, W.L. New similarity measures of intuitionistic fuzzy sets based on the Jaccard index with its application to clustering. Int. J. Intell. Syst. 2018, 33, 1672-1688. [CrossRef]

4. Wang, Z.; Xu, Z.; Liu, S.; Yao, Z. Direct clustering analysis based on intuitionistic fuzzy implication. Appl. Soft Comput. 2014, 23, 1-8. [CrossRef]

5. Xu, D.; Xu, Z.; Liu, S.; Zhao, H. A spectral clustering algorithm based on intuitionistic fuzzy information. Knowl. Based Syst. 2013, 53, 20-26. [CrossRef]

6. Garg, H.; Kumar, K. An advanced study on the similarity measures of intuitionistic fuzzy, sets based on the set pair analysis theory and their application in decision making. Soft Comput. 2018, 22, 4959-4970. [CrossRef]

7. He, Y.; Chen, H.; Zhou, L.; Liu, J.; Tao, Z. Intuitionistic fuzzy geometric interaction averaging operators and their application to multi-criteria decision making. Inf. Sci. 2014, 269, 142-159. [CrossRef]

8. Liu, F.; Aiwu, G.; Lukovac, V.; Vukic, M. A multicriteria model for the selection of the transport service provider: A single valued neutrosophic DEMATEL multicriteria model. Dec. Mak. Appl. Manag. Eng. 2018, 1, 121-130. [CrossRef]

9. Pedrycz, W.; Chen, S.M. Granular Computing and Decision-Making: Interactive and Iterative Approaches; Springer: Berlin, Germany, 2015.

10. Stanujkić, D.; Karabašević, D. An extension of the WASPAS method for decision-making problems with intuitionistic fuzzy numbers: A case of website evaluation. Oper. Res. Eng. Sci. Theo. Appl. 2018, 1, 29-39. [CrossRef] 
11. Wang, J.Q.; Zhang, H.Y. Multicriteria decision-making approach based on Atanassov's intuitionistic fuzzy sets with incomplete certain information on weights. IEEE Trans. Fuzzy Syst. 2013, 2, 510-515. [CrossRef]

12. De, S.K.; Biswas, R.; Roy, A.R. An application of intuitionistic fuzzy sets in medical diagnosis. Fuzzy Sets Syst. 2001, 117, 209-213. [CrossRef]

13. Papacostas, G.A.; Hatzimichaillidis, A.G.; Kaburlasos, V.G. Distance and similarity measures between intuitionistic fuzzy sets: A comparative analysis from a pattern recognition point view. Pattern Recognit. Lett. 2013, 34, 1609-1622. [CrossRef]

14. Boran, F.E.; Akay, D. A biparametric similarity measure on intuitionistic fuzzy sets with applications to pattern recognition. Inf. Sci. 2014, 255, 45-57. [CrossRef]

15. Chen, S.M.; Chang, C.H. A novel similarity measure between Atanassov's intuitionistic fuzzy sets based on transformation techniques with applications to pattern recognition. Inf. Sci. 2015, 291, 96-114. [CrossRef]

16. Chen, S.M.; Randyanto, Y. A novel similarity measure between intuitionistic fuzzy sets and its applications. Int. J. Pattern Recognit. Artif. Intell. 2013, 27, 1350021. [CrossRef]

17. Mitchell, H.B. On the Dengfeng-Chuntian similarity measure and its application to pattern recognition. Pattern Recognit. Lett. 2003, 24, 3101-3104. [CrossRef]

18. Qian, J.; Xin, J.; Lee, S.J.; Yao, S. A new similarity/distance measure between intuitionistic fuzzy sets based on the transformed isosceles triangles and its applications to pattern recognition. Expert Syst. Appl. 2019, 116, 439-453.

19. Ye, J. Cosine similarity measures for intuitionistic fuzzy sets and their applications. Math. Comput. Model. 2011, 53, 91-97. [CrossRef]

20. Beliakov, G.; Pagola, M.; Wilkin, T. Vector valued similarity measures for Atanassov's intuitionistic fuzzy sets. Inf. Sci. 2014, 280, 352-367. [CrossRef]

21. Chen, S.M. Similarity measures between vague sets and between elements. IEEE Trans. Syst. Man Cybern. 1997, 27, 153-158. [CrossRef]

22. Chen, S.M.; Cheng, S.H.; Lan, T.C. A novel similarity measure between intuitionistic fuzzy sets based on the centroid points of transformed fuzzy numbers with applications to pattern recognition. Inf. Sci. 2016, 343, 15-40. [CrossRef]

23. Dengfeng, L.; Chuntian, C. New similarity measures of intuitionistic fuzzy sets and application to pattern recognitions. Pattern Recognit. Lett. 2002, 23, 221-226. [CrossRef]

24. Li, F.; Xu, Z. Similarity measures between vague sets. J. Softw. 2001, 12, 922-927.

25. Grzegorzewski, P. Distances between intuitionistic fuzzy sets and/or interval-valued fuzzy sets based on the Hausdorff metric. Fuzzy Sets Syst. 2004, 148, 319-328. [CrossRef]

26. Hong, D.H.; Kim, C. A note on similarity measures between vague sets and between elements. Inf. Sci. 1999, 115, 83-96. [CrossRef]

27. Hung, W.L.; Yang, M.S. Similarity measures of intuitionistic fuzzy sets based on Hausdorff distance. Pattern Recognit. Lett. 2004, 26, 1603-1611. [CrossRef]

28. Li, Y.; Olson, D.L.; Qin, Z. Similarity measures between intuitionistic fuzzy (vague) sets: A comparative analysis. Pattern Recognit. Lett. 2007, 28, 278-285. [CrossRef]

29. Li, Y.; Zhongxian, C.; Degin, Y. Similarity measures between vague sets and vague entropy. J. Comput. Sci. 2002, 29, 129-132. (In Chinese)

30. Liang, Z.; Shi, P. Similarity measures on intuitionistic fuzzy sets. Pattern Recognit. Lett. 2003, 24, $2687-2693$. [CrossRef]

31. Atanassov, K. On Intuitionistic Fuzzy Sets Theory; Springer: Berlin, Germany, 2012.

32. Atanassov, K. Intuitionistic Fuzzy Sets; Springer: Heidelberg, Germany, 1999.

33. Szmidt, E.; Kacprzyk, J. Distances between intuitionistic fuzzy sets. Fuzzy Sets Syst. 2000, 114, 505-518. [CrossRef]

(C) 2019 by the authors. Licensee MDPI, Basel, Switzerland. This article is an open access article distributed under the terms and conditions of the Creative Commons Attribution (CC BY) license (http://creativecommons.org/licenses/by/4.0/). 